\title{
Investigation of Carrier Recombination in Si Heavily Irradiated by Neutrons
}

\author{
E. Gaubas*, A. Kadys, A. Uleckas and J. Vaitkus \\ Vilnius University, Institute of Materials Science and Applied Research \\ Saulètekio al. 10, LT-10223 Vilnius, Lithuania \\ Variations of recombination lifetime, with fluence of the reactor neu- \\ trons from $10^{12}$ to $3 \times 10^{16} \mathrm{n} / \mathrm{cm}^{2}$, in the magnetic field applied Czochralski \\ grown $\mathrm{Si}$ samples are examined by the contactless transient techniques of \\ the microwave probed photoconductivity and dynamic gratings. A nearly \\ linear decrease in lifetime from few microseconds to about 200 ps within the \\ examined range of neutron irradiation fluences was obtained. This depen- \\ dence persists under relatively low $\left(\leq 80^{\circ} \mathrm{C}\right)$ temperature heat treatments. \\ Also, cross-sectional scans of lifetime depth-profiles were examined, which \\ show rather high homogeneity of lifetime values within wafer thickness.
}

PACS numbers: 61.72.J-, 61.82.Fk, 72.40.+w

\section{Introduction}

The modern techniques based on semiconductor devices need materials with high switching rates, high frequencies, and fast recovery. These parameters can be achieved by manipulating carrier lifetime, which is very sensitive to the structure and density of crystal defects. On the other hand, to increase charge collection efficiency in detector materials, carrier lifetime should be maintained rather long. In this work, recombination lifetime manipulations via introduction of radiation defects by neutron irradiation and post-irradiation heat treatments are investigated.

\section{Samples and experimental techniques}

High purity, Czochralski grown under magnetic field (MCZ) Si wafers and diode structures of the CERN standard particle detectors have been investigated. These samples were irradiated by reactor neutrons using different fluences in the range from $10^{12}$ to $3 \times 10^{16} \mathrm{n} / \mathrm{cm}^{2}$. Carrier recombination lifetime has been directly measured in the as-irradiated samples, kept under $10^{\circ} \mathrm{C}$ temperature, and

*corresponding author; e-mail: eugenijus.gaubas@ff..vu.lt 
annealed after irradiations at $80^{\circ} \mathrm{C}$ for $5 \mathrm{~min}$ as well as $30 \mathrm{~min}$ and $24 \mathrm{~h}$. Recombination characteristics have been examined by exploiting excess carrier decay transients measured by combining the microwave probed photoconductivity (MWPC) [1] and dynamic grating (DG) [2] techniques. The fluence dependent carrier lifetime variations have been analyzed by comparing with those measured in proton irradiated high resisitivity, float-zone (FZ) grown Si material.

\section{Fluence and heat treatment dependent recombination lifetime variations}

Lifetime variation with neutron fluence measured in the as-irradiated and kept in freezer at $10^{\circ} \mathrm{C}$ wafer samples is shown in Fig. 1a. These values have been measured by combining MW-PC and DG techniques when lifetime is directly extracted from excess carrier density relaxation rate or from MW-PC amplitude decrease and using DG grating erase time dependence on grating spacing in the range of short lifetimes $[1,2]$. Lifetime values determined by DG and MW-PC correlate well when measured for the same fluence irradiated samples. MW-PC transients exhibit one-componential relaxation indicating prevalence of recombination process. A decrease of recombination lifetime is close to linear relatively to enhancement of irradiation fluence.

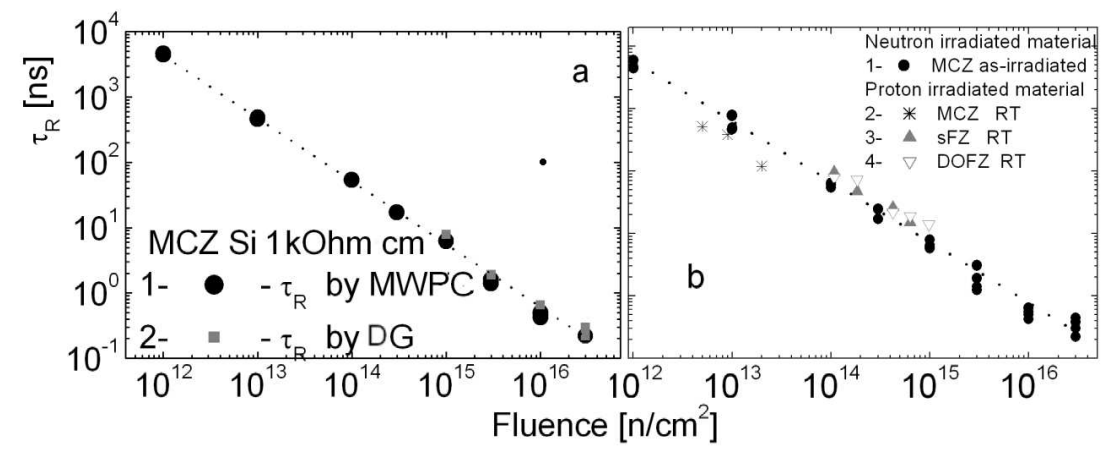

Fig. 1. Recombination lifetime as a function of neutron irradiation fluence in MCZ Si wafers: (a) evaluated by MW-PC (1) and DG (2) transient techniques. (b) Recombination lifetime obtained in MCZ Si as-irradiated (1) wafer and diode structures compared with lifetime values measured in MCZ Si diodes (2), and (3) FZ Si CERN standard (sFZ) as well as (4) DOFZ Si particle detectors irradiated with high energy protons and long stored at RT.

Values of recombination lifetime obtained in wafer samples nearly coincide with those measured in diode structures irradiated and kept at the same conditions, as illustrated in Fig. 1b. Moreover, the lifetime dependence on fluence measured in neutron irradiated wafer and detector (MCZ Si based) structures is close to that determined for proton irradiated standard FZ and diffusion oxygenated FZ 
(DOFZ) Si diodes, as shown in Fig. 1b. Congruity of these dependences indicates that high-energy protons and neutrons induce the same prevailing recombination centers in both MCZ and FZ grown materials.

The recombination lifetime values in the irradiated structures are slightly varied under long storage at room temperature (RT). The lifetime values can be increased using anneals at elevated temperatures, as shown in Fig. 2a for MCZ wafers, heat treated at $80^{\circ} \mathrm{C}$ temperature using different exposures. Lifetime values can be nearly doubled after prolonged anneal at elevated temperature. Transformations of defects can be a reason for small deviations of lifetime values obtained for FZ Si proton irradiated diodes, long kept at RT, from those measured in MCZ material kept in freezer (a comparison is shown in Fig. 1b).
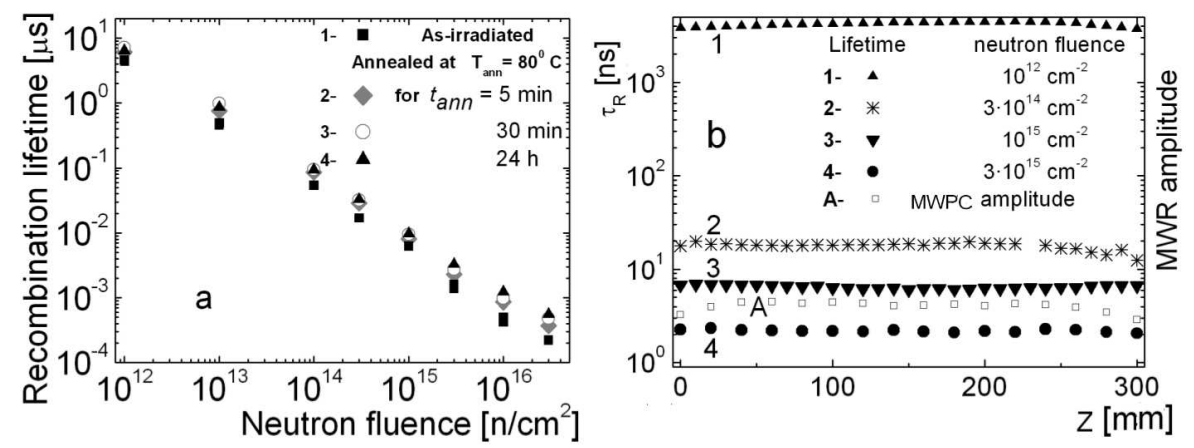

Fig. 2. (a) Fluence dependent lifetime variations in MCZ Si under anneal regimes: as-irradiated (1), and annealed at $80^{\circ} \mathrm{C}$ for $5 \mathrm{~min}(2), 30 \mathrm{~min}(3)$ and $24 \mathrm{~h}$ (4). (b) Recombination lifetime within wafer cross-section of MCZ Si wafers irradiated by different fluences of reactor neutrons: $10^{12}(1), 3 \times 10^{14}(2), 10^{15}(3)$, and $3 \times 10^{15} \mathrm{n} / \mathrm{cm}^{2}(4)$, and MW-PC amplitude $(A)$.

High-energy neutrons introduce rather homogeneous distribution of recombination centers within thickness of wafer samples irrespective of irradiation fluence, as illustrated in Fig. 2b. These lifetime profiles within wafer thickness have been controlled by lifetime cross-sectional scans performed by MW-PC technique exploiting a sharply focused laser beam and near-field needle-tip MW antenna. In these experiments, a sample is translated relatively to the intersection point of the MW antenna - laser beam within wafer cross-section by moving a microstep stage. While, an amplitude of the MW-PC response is more sensitive to boundary effects, when decrease in excess carrier density due to surface recombination and less excitation intensity can be observed within the photoresponse profile (curve $A$ in Fig. 2b).

Short lifetimes obtained in the range of the largest fluences and nearly linear reduction of recombination lifetime with fluence enhancement imply dominance of the recombination centers characterized by a large capture cross-section. It 
is well known [3] from simulations of the high-energy particle interactions with material that hadrons, especially neutrons, introduce cluster type extended defects. The large capture cross-sections can be assumed for the extended clusters, to explain short and rather stable values of carrier recombination lifetime observed in our experiments. Existence of clusters is corroborated by combined study of more specific characteristics of these samples, such as DG diffraction efficiency, the amplitudes and shape of the MW-PC transients, as well as carrier scattering parameters. Then, rather moderate lifetime variations under anneals of irradiated samples can be qualitatively understood by transformations of the surrounding complexes, which compose these clusters, while the whole cluster remains recombination active.

In summary, a linear decrease in the carrier recombination lifetime from few microseconds to hundreds of picoseconds with enhancement of fluence of the high-energy neutrons and protons in the range from $10^{12}$ to $3 \times 10^{16} \mathrm{~cm}^{-2}$ has been obtained for MCZ and FZ Si structures. Manipulation of lifetime values by neutrons fluence can be applied for improvement of Si device switching rates. Homogeneous lifetime distribution within depth of the Si structures irradiated by reactor neutrons has been revealed. The observed recombination characteristics can be qualitatively explained by formation of the cluster-type recombination centers.

\section{Acknowledgments}

These investigations were performed within CERN rd50-WODEAN project and partly supported by Lithuanian State Science and Studies Foundation.

\section{References}

[1] E. Gaubas, Lith. J. Phys. 43, 145 (2003).

[2] K. Jarasiunas, J. Vaitkus, E. Gaubas, L. Jonikas, R. Pranaitis, L. Subacius, IEEE J. QE QE-22, 1298 (1986).

[3] C. Claeys, E. Simoen, Radiation Effects in Advanced Semiconductor Materials and Device, Springer, Berlin 2002.

Page 2 - Section 3, first paragraph, lines $9-10$ : the sentence "Recombination lifetime" might be edited in such a manner - " " - In the legend of Fig. 1a should be "MW-PC" instead of "MWR" and "DG" instead of "TG" - Caption of figure 1: should be "MW-PC" instead of "MW-PCD" Page 3 - In the legend of Fig. 2b should be " A - MW-PC amplitude" instead of "A - MWR amplitude" - Label at X-axis of Fig. $2 \mathrm{~b}$. should be " Z [mm]" instead of " Z [ms]" 